\title{
Theoretical Study of Need for Nurses Support in Early Initiation of Breastfeeding
}

\section{Oparanma FU*}

Department of Nursing, Rivers State University, Nigeria

\section{Review Article}

Volume 3 Issue 5

Received Date: August 06, 2019

Published Date: September 13, 2019

DOI: $10.23880 /$ nhij-16000200

University, Nigeria, Email: okachi@yahoo.com

\section{Abstract}

The purpose of this theoretical study was to investigate or x-ray the vital roles nurses should play to support early initiation of breastfeeding and its impact within the first hour of birth. Breastfeeding within the first hour of life is associated with better outcomes like exclusive breastfeeding and duration. Nurses' support is crucial and lack of support from healthcare professionals like nurses has been linked to delayed initiation of breastfeeding and poor outcome. Therefore, we concluded that it is imperative as a cardinal duty for the nurses to recommend and if possible to ensure early intervention.

Keywords: Early initiation; Breastfeeding; Nurse

\section{Introduction}

Breastfeeding has many benefits to both infants and mothers. Despite the numerous advantages of breastfeeding, the initiation and duration rates for breastfeeding is still relatively low. In Nigeria, up to $75 \%$ of mothers do decide to breastfeed their babies; however, only $13 \%$ are exclusively breastfeeding at 6 months and $22 \%$ continuing breastfeeding at 1 year. Our nation's public health priorities identified by Healthy People 2020 includes targets for breastfeeding initiation at 81.9\%, 6 months exclusive breastfeeding at $25.5 \%$, and $34.1 \%$ continuing breastfeeding at 1 year [1]. Studies have cited that the most important influence on breastfeeding behaviors and intentions is breastfeeding help within half an hour of birth and that those who received this support had better breastfeeding outcomes not only in the short term but also once the women had left the hospital, with an improved rate of breastfeeding exclusively at two weeks. No study that we know of has been done on nurses' perceptions, beliefs and attitudes about breastfeeding to determine if it has any effect on providing breastfeeding support to women particularly within the first hour after birth.

\section{Significance of the Early Initiation}

Several studies have highlighted the importance of breastfeeding to both infants and mothers. The benefits of breastfeeding are both psychological, as it facilitates bonding between mother and child, and physical, as it reduces rates of otitis media, gastrointestinal disease, respiratory disease, asthma and some maternal cancer [2]. According to this study, the importance of professional support for breastfeeding has been recognized in an evidenced-based breastfeeding policy, the World Health Organization (WHO) and United Nations International Children's Fund (UNICEF) Baby Friendly Hospital Initiative. Ten steps of the Baby Friendly Hospital Initiative strive to promote successful breastfeeding with the fourth step calling to help mothers initiate breastfeeding within one half-hour of birth. Mothers who breastfed within the first hour of birth are more likely to be exclusively breastfeeding two to four weeks after discharge [3]. Many factors have been cited in multiple studies that contribute to poor breastfeeding outcomes including delayed initiation, duration, and exclusive breastfeeding. Support from healthcare professionals 


\section{Nursing \& Healthcare International Journal}

have been identified as the most significant. The most important influence on breastfeeding behaviors and intentions is breastfeeding help within half an hour of birth. Those who received this support had better breastfeeding outcomes not only in the short term but also once the women had left the hospital, with an improved rate of breastfeeding exclusively at two weeks [2]. There is the need to understand why breastfeeding support by healthcare providers is poor and the barriers for them in providing the needed support to women. This study will contribute in formulating interventions that will help improve early initiation, rate and duration of breastfeeding. The knowledge and attitudes of health professionals are important factors in the promotion and support of breastfeeding [4].

\section{Theoretical framework}

The theoretical framework for this study was the Theory of Reasoned Action (TRA) by Fishbein and Ajzen, which studies the relationship between behavior and attitudes. This theory suggests that a confluence of social and personal beliefs regarding a particular behavior determines one's intention to perform, or not perform, a given behavior [5]. A person's attitudes and beliefs about a particular behavior are developed based on prior knowledge and experiences relative to the behavior, and their social pressures (subjective norms) to perform or not perform that behavior. These attitudes and beliefs are directly related to their intentions toward the behavior [6]. The concepts that form the basis of the TRA include behaviors, intentions, attitudes, subjective norms, and perceived self-control. The theory, which specifies the nature of the relationship among these concepts, provides a framework for generating hypothesis relating to health behaviors [7]. The researcher hypothesized that the support of nurses regarding breastfeeding will determine their behavior to or not to support and encourages the initiation of breastfeeding immediately after birth.

\section{Review of Literature}

Many leading health organizations, including the World Health Organization (WHO), the American Academy of Pediatrics, and the United Nations Children's Fund (UNICEF), recommends breastfeeding as the best nutrition for infant and that breastfeeding confer numerous health benefits to both infant and mother. The benefits of breastfeeding and breast milk has been supported by numerous research studies, [1-3,6,8-10]. Despite the numerous advantages of breastfeeding, the initiation and duration rates for breastfeeding is still relatively low. In Nigeria, $75 \%$ of mothers do decide to breastfeed their babies; however, only $13 \%$ are exclusively breastfeeding at 6 months and $22 \%$ continuing breastfeeding at 1 year. Our nation's public health priorities identified by Healthy People 2020 includes targets for breastfeeding initiation at 81.9\%, 6 months exclusive breastfeeding at 25.5\%, and $34.1 \%$ continuing breastfeeding at 1 year [1].

Early initiation of breastfeeding especially within the first hour of birth is associated with better breastfeeding outcomes including duration and exclusive breastfeeding. The WHO and UNICEF Baby Friendly Hospital Initiative Ten Steps to Successful breastfeeding also highlighted the importance of early initiation of breastfeeding in step four asking health professionals to help mothers initiate breastfeeding within one half-hour of birth. The World Health Organization summarized the findings of studies on the effects of early breastfeeding contact in Step 4 of Evidence for The Ten Steps to Successful Breastfeeding which states "Help mothers initiate breastfeeding within a half-hour of birth" [11]. Research on the benefits of early commencement of breastfeeding has been well documented [2,6,8,10-12]. Breastfeeding within the first hour of life is associated with better breastfeeding outcomes like exclusive breastfeeding and duration [3].

Breastfeeding outcomes have also been linked to the support women receive from healthcare professionals during the immediate period after delivery. Nurses are considered to be the ones who maintain, encourage, and support breastfeeding [8]. The health professionals a woman encounters in the antenatal and postnatal periods can influence her positively or negatively in her decision to breastfeed or to continue breastfeeding [4]. A study by Kervin, et al. [2], shows that support is effective in increasing intention, initiation, duration and exclusivity of breastfeeding, and improves maternal confidence. The study went on to say that support is most effective when delivered in both the pre-and post-natal period and can take many formats. Many factors affect the initiation and continuation of breastfeeding but the quality of the breastfeeding instruction and support the mother receives during the immediate hospital postpartum period is an important influencing factor [6]. Despite the effectiveness of professional support interventions, access to these sources of support remains very low and these professional support practices were not reported by most women, which may contribute to the poor breastfeeding outcomes [2].

The lack of support and encouragement from healthcare professionals like nurses is also closely related to breastfeeding outcomes. In an effort to improve breastfeeding outcomes, there is the need to understand 


\section{Nursing \& Healthcare International Journal}

why professional support practices from nurses are lacking. Health professionals like nurses are an important group to consider when trying to improve the standard of support available to breastfeeding women and therefore need good knowledge and a positive attitude towards breastfeeding [4]. So far, several studies have been published about the benefits of breastfeeding, factors that affect breastfeeding outcomes both positively and negatively, and several interventions to improve breastfeeding outcomes, but no study is available on the impact of support about breastfeeding on breastfeeding outcomes particularly the initiation of breastfeeding within the first hour after delivery. Any effort to increase rates of breastfeeding must take into account the knowledge and support of healthcare professionals and also their training needs [4].

\section{Summary}

The aim of this study was to investigate nurse's support about breastfeeding and its influence in the support and encouragement they provide to new moms regarding breastfeeding initiation immediately after delivery. This study went on to say that breastfeeding behavior within the first hour was significantly better among those mothers who received positive breastfeeding supports from their health-care team and many women who did not start or had very limited period of breastfeeding was due to lack of healthcare team support of breastfeeding and unsupportive attitudes.

\section{Conclusion}

In this study the researcher was able to conclude that the importance of early breast feeding support with the help of health professionals like nurses cannot be overemphasized. The study had shown that there are some important gains of early support in breast feeding and mothers know some of these gains as they apply to the growing process of infants. This is in agreement with WHO, UNICEF and FNRI. (food and nutrition research institute) that recommends that infants should be fed very early with the support of the health care providers.

\section{References}

1. Stanton R (2011) A road map for change: Ensuring that women have breastfeeding support. J Perinat Educ 20(3): 130-133.

2. Kervin BE, Kemp L, Pulver LJ (2010) Types and timing of breastfeeding support and its impact on mothers' behaviours. J Paediatr Child Health 46(3): 85-91.
3. Difrisco E, Goodman KE, Budin WC, Lilienthal MW, Kleinman A, et al. (2011) Factors associated with exclusive breastfeeding 2 to 4 weeks following discharge from a large, urban, academic medical center striving for Baby-Friendly designation. J Perinat Educ 20(1): 28-35.

4. Whelan B, McEvoy S, Eldin N, Kearney J (2011) What primary health professionals need to promote breastfeeding. Practice Nursing 22(1): 35-39.

5. Pickett MC (2011) Theory of reasoned action: Reassessing the relationships of moral and ethical climates in organizations. Journal of Organizational Psychology 11: 63-72.

6. Bernaix LW, Beaman ML, Schmidt CA, Harris JK, Miller LM (2010) Success of an educational intervention on maternal/newborn nurses' breastfeeding knowledge and attitudes. Journal of Obstetric, Gynecologic, and Neonatal Nursing 39: 658-666.

7. Polit DF, Beck CT (2012) Nursing research: Generating and assessing evidence for nursing practice $9^{\text {th }}(\mathrm{edn}$.$) , Philadelphia: Lippincott Williams \&$ Wilkins.

8. Duran S, Duran R, Sahin EM, Dagdeviren N, Guzel A (2010) Comparison of the knowledge and attitudes of midwives about breastfeeding with breastfeeding behaviors of the mothers they provide counseling. Balkan Medical Journal 27(1): 11-17.

9. Manganaro $\mathrm{R}$, Marseglia L, Mamì $\mathrm{C}$, Paolata $\mathrm{A}$, Gargano R (2008) Effects of hospital policies and practices on initiation and duration of breastfeeding. Child: Care, Health and Development 35(1): 106-111.

10. Watkins AL, Dodgson JE (2010) Breastfeeding educational interventions for health professionals: A synthesis of intervention studies. J Spec Pediatr Nurs 15(3): 223-232.

11. Nakao Y, Moji K, Honda S, Oishi K (2008) Initiation of breastfeeding within 120 minutes after birth is associated with breastfeeding at four months among Japanese women: A self-administered questionnaire survey. Int Breastfeed J 3(1): 1-7.

12. Vieira TO, Vieira GO, Giugliani ER, Mendes CM, Martins CC (2010) Determinants of breastfeeding initiation within the first hour of life in Brazilian population: Cross-sectional study. BMC Public Health 10: 760 .

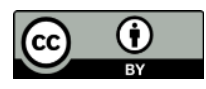

